\title{
Effect of Shengjin Pingchuan Decoction Combined with Qihuang Granule on the Improvement of Cerebral Bronchiolitis Symptoms and Recurrence Rate in Children
}

\author{
Shuling Wang, Jianghong Hou, Tuanying Chen, Dongdong Shen \\ Henan Province Hospital of Traditional Chinese Medicine, Henan, Zhengzhou 450002
}

Keywords: Pediatric Bronchiolitis; Qihuang Granules; Shengjin Pingchuan Decoction; symptom improvement; recurrence rate

\begin{abstract}
Objective: To investigate the value of Shengjin Pingchuan Decoction and Qihuang Granule in the treatment of children with bronchiolitis. Methods: From July 2016 to October 2017, 106 children with bronchiolitis were enrolled in our hospital. According to the random number table method, the observation group and the control group were divided into 53 groups. On the basis of conventional treatment, the control group was treated with Qihuang Granules, and the observation group was treated with Qihuang Granule and Shengjin Pingchuan Decoction. Both groups were treated continuously for 7 days. The clinical efficacy, symptoms and signs of improvement and the changes of inflammatory index [TNF- $\alpha$ ] levels before and after treatment were compared between the two groups. The patients were followed up for 6 months after the end of treatment, and the recurrence rate of the two groups was counted. Results: The total effective rate of the observation group was $92.45 \%$ (49/53), which was higher than that of the control group (77.36\%, 41/53, $\mathrm{P}<0.05$ ). The observation group had fever subsided, cough improved, wheezing relief, lung wet rales and wheezing sounds. The disappearance time was shorter than that of the control group $(\mathrm{P}<0.05)$. The levels of TNF- $\alpha$ in the two groups were lower than those before treatment $(\mathrm{P}<0.05)$, and the observation group was lower than the control group $(\mathrm{P}<0.05)$. The disease recurrence rate was $3.77 \%$ in the observation group. (2/53) was lower than the control group by $15.09 \%(8 / 53$, $\mathrm{P}<0.05)$. Conclusion: Shengjin Pingchuan Decoction combined with Qihuang Granule in the treatment of children with bronchiolitis is effective, which can effectively promote the relief of symptoms and signs, may be related to the down-regulation of serum inflammatory factor expression, and also help to reduce the recurrence rate of the disease.
\end{abstract}

\section{Introduction}

Bronchiolitis is a common acute lower respiratory tract infection in pediatrics. Due to the poor treatment of conventional western medicine and obvious side effects, the clinical treatment of pediatric bronchiolitis has gradually turned to the research direction of traditional Chinese medicine in recent years [1]. Bronchiolitis is a category of traditional Chinese medicine "pneumonia, asthma, cough" and "hepatitis syndrome", and Chinese medicine believes that children's lungs are delicate and delicate, and they are susceptible to wind and evil, resulting in lack of righteousness and qi and yin deficiency, which in turn induces this disease [2]. Qihuang granule is a proprietary Chinese medicine with scorpion, medlar and huangjing as the main components. It has the effect of moistening heart and lung and replenishing vital energy. It is gradually recognized in the treatment of pediatric bronchiolitis. In addition, Chinese medicine believes that spleen and lung qi deficiency, treatment of the cultivation of soil and gold, Xuanfei Huayu, and Shengjin Pingchuan Tang just have the above functions, it is expected to achieve a certain effect in the treatment of asthma caused by spleen and lung qi deficiency. However, there is no research report on the treatment of children with bronchiolitis with Shengjin Pingchuan Decoction combined with Qihuang Granule. In this study, 106 children with bronchiolitis in our hospital were selected to discuss the clinical application value of the above-mentioned TCM combination regimen. 


\section{Materials and Methods}

\subsection{General Information.}

From July 2016 to October 2017, 106 children with bronchiolitis in our hospital were selected and divided into observation group and control group according to the random number table method. There were 53 patients in the observation group, including 27 males and 26 females, aged from 8 months to 3 years old, with an average of $(1.87 \pm 0.70)$ years; the course of disease was 1 to 5 days, with an average of $(2.99 \pm 1.12)$ days. There were 53 patients in the control group, including 25 males and 28 females, aged from 7 months to 3 years, with an average of $(1.91 \pm 0.67)$ years; the course of disease was 1 to 6 days, with an average of $(3.02 \pm 1.08)$ days. There was no significant difference in baseline data between the two groups $(\mathrm{P}>0.05)$. This study meets the relevant requirements of the Helsinki Declaration of the World Medical Association and is approved by the hospital ethics committee.

\subsection{Case Entry Criteria.}

Inclusion criteria: (1) Comply with the diagnostic criteria for bronchiolitis in Zhufu Practical Pediatrics [3]; (2) are the first onset; (3) informed consent of the family members of the study. Exclusion criteria: (1) combined with lung infection, bronchial asthma, tuberculosis and other diseases; (2) liver, kidney and other important organ dysfunction or congenital heart disease; (3) allergies; (4) poor compliance, can not Oral Chinese medicine.

\subsection{Methods.}

Treatment Methods Both groups were given conventional oxygen therapy, anti-infection, antispasmodic, anti-inhalation, aerosol inhalation, correction of acid-base imbalance and water-electrolyte disorders. On the basis of this, (1) the control group was given Qihuang granules. (2) The observation group was treated with Shengjin Pingchuan Decoction on the basis of the control group. The basic prescription consisted of: $15 \mathrm{~g}$ of Radix Pseudostellariae, $15 \mathrm{~g}$ of Atractylodes Rhizome, $6 \mathrm{~g}$ of dried tangerine peel, $6 \mathrm{~g}$ of Pinellia ternata, $6 \mathrm{~g}$ of Pinellia ternata, $6 \mathrm{~g}$ of Astragalus membranaceus, $6 \mathrm{~g}$ of Ginger, and $5 \mathrm{~g}$ of ginger. , Aster $4 \mathrm{~g}$, Campanulaceae $2 \mathrm{~g}$, mustard 2 g, almond 2 g, coltsfoot 2 g, shot 2 g, ephedra 2 g; 1 dose / d, water decoction juice 50 $80 \mathrm{ml}, 2$ times in the morning and evening clothes. Both groups were treated continuously for 7 days.

Serum inflammatory index test method Take $2 \mathrm{ml}$ of venous blood in the fasting state before and after treatment in the morning, centrifuge at $2500 \mathrm{r} / \mathrm{min}$ for $15 \mathrm{~min}$, take the upper serum for examination, and detect serum tumor necrosis factor by enzyme-linked immunosorbent assay. The $\alpha$ (TNF- $\alpha$ ) level was obtained by Bio-RAD Bio-RAD550 type microplate reader from USA, and the kit was purchased from Guangzhou Wanfu Biotechnology Co., Ltd.

\subsection{Efficacy Evaluation Criteria.}

According to the "Diagnostic and therapeutic criteria for clinical symptoms of traditional Chinese medicine" [4], the efficacy is effective, effective, ineffective, total effective rate = (effective + effective) / total number of cases $\times 100 \%$. After 3 days of treatment, the cough was relieved, the wheezing was relieved, the lung wet rales and wheezing sound disappeared or significantly reduced to be markedly effective; after 4 to 7 days of treatment, cough reduction, wheezing relief, lung wet rales and wheezing disappeared or Significant reduction was effective; after treatment for more than 7 days, the symptoms showed no obvious relief or aggravation, severe asthma, and the need for intravenous corticosteroids was ineffective.

\subsection{Observation Indicators.}

(1) Clinical efficacy. (2) Improvement of symptoms and signs. The records were compared between the two groups: fever subsidence, cough improvement, wheezing relief, and lung wet rales and wheezing sound disappeared. (3) Changes in inflammatory indicators. Changes in serum TNF- $\alpha$ levels before and after treatment. (4) Recurrence rate. After 6 months of follow-up treatment, the 
recurrence rate of the two groups was statistically compared.

Statistical analysis Statistical analysis by SPSS18.0 software, measurement data $( \pm \mathrm{s})$, $\mathrm{t}$ test, count data $\mathrm{n}(\%), \chi^{2}$ test, $\mathrm{P}<0.05$ for the difference was statistically significant.

\section{Results}

\subsection{Clinical Efficacy.}

The total effective rate of the observation group was $92.45 \%$ higher than that of the control group (77.36\%) $(\mathrm{P}<0.05)$, as shown in Table 1.

Table 1 Comparison of clinical effects between the two groups [n (\%)]

\begin{tabular}{llllll}
\hline Group & $\begin{array}{l}\text { Number } \\
\text { of cases }\end{array}$ & $\begin{array}{l}\text { Significant } \\
\text { effect }\end{array}$ & effective & invalid & $\begin{array}{l}\text { Total } \\
\text { efficiency }\end{array}$ \\
\hline $\begin{array}{l}\text { Observation } \\
\text { group }\end{array}$ & 53 & 27(50.94) & 22(41.51) & $4(7.55)$ & $49(92.45)$ \\
$\begin{array}{l}\text { Control } \\
\text { group }\end{array}$ & 53 & $20(37.74)$ & 21(39.62) & 12(22.64) & $41(77.36)$ \\
$\chi^{2}$ & & & & & 4.711 \\
$P$ & & & & & 0.030 \\
\hline
\end{tabular}

Symptoms and signs improved in the observation group The fever disappeared, cough improved, wheezing relief, lung wet rales and wheezing disappeared shorter than the control group $(\mathrm{P}<0.05)$, see Table 2.

Table 2 Comparison of symptoms and signs improvement between the two groups ( \pm s, d)

\begin{tabular}{llllllll}
\hline Group & $\begin{array}{l}\text { Number } \\
\text { of cases }\end{array}$ & $\begin{array}{l}\text { Fever } \\
\text { subsided }\end{array}$ & $\begin{array}{l}\text { Cough } \\
\text { getting } \\
\text { better }\end{array}$ & $\begin{array}{l}\text { Asthma } \\
\text { relief }\end{array}$ & $\begin{array}{l}\text { Wet rales } \\
\text { disappear }\end{array}$ & $\begin{array}{l}\text { Wheezing } \\
\text { disappears }\end{array}$ \\
\hline $\begin{array}{l}\text { Observati } \\
\text { on group }\end{array}$ & 53 & $2.29 \pm 1.24$ & $4.12 \pm 1.36$ & $3.97 \pm 1.84$ & $5.77 \pm 2.03$ & $5.01 \pm 2.14$ \\
$\begin{array}{l}\text { Control } \\
\text { group }\end{array}$ & 53 & $3.08 \pm 1.31$ & $5.17 \pm 1.53$ & $5.06 \pm 1.23$ & $6.85 \pm 2.12$ & $6.16 \pm 2.25$ \\
$t$ & & 3.188 & 3.734 & 3.585 & 2.679 & 2.696 \\
$P$ & & 0.002 & 0.000 & 0.001 & 0.009 & 0.008 \\
\hline
\end{tabular}

Serum TNF- $\alpha$ level There was no significant difference in serum TNF- $\alpha$ levels between the two groups before treatment $(\mathrm{P}>0.05)$. After treatment, the levels of TNF- $\alpha$ in the two groups were lower than those before treatment $(\mathrm{P}<0.05)$, and the observation group was lower than the control group. $(\mathrm{P}<0.05)$, see Table 3 .

Table 3 Comparison of serum TNF- $\alpha$ levels before and after treatment in both groups $( \pm \mathrm{s}, \mathrm{ng} / \mathrm{ml})$

\begin{tabular}{llllll}
\hline Group & $\begin{array}{l}\text { Number } \\
\text { of cases }\end{array}$ & $\begin{array}{l}\text { Before } \\
\text { treatment }\end{array}$ & $\begin{array}{l}\text { After } \\
\text { treatment }\end{array}$ & $t$ & $P$ \\
\hline $\begin{array}{l}\text { Observation } \\
\text { group }\end{array}$ & 53 & $2.73 \pm 0.88$ & $1.02 \pm 0.62$ & 11.565 & 0.000 \\
Control & 53 & $2.69 \pm 0.91$ & $1.37 \pm 0.75$ & 8.149 & 0.000 \\
group & & 0.230 & 2.619 & & \\
$t$ & & 0.819 & 0.010 & & \\
$P$ & & &
\end{tabular}

\subsection{Recurrence Rate.}

After 6 months of treatment, the recurrence rate was 3.77\% (2/53) in the observation group and 8 cases in the control group. The recurrence rate was $15.09 \%$ (8/53). Statistical significance 
$(\chi 2=3.975, \mathrm{P}=0.046)$.

\section{Discussion}

Bronchiolitis is a common and serious acute lower respiratory tract infection in children. Inflammation can involve alveolar, pulmonary interstitial and alveolar walls, so it is also a special type of pneumonia. Western medicine treatment of children with bronchiolitis to control infection and relieve symptomatic asthma, cough and phlegm and other symptomatic treatment, although it can relieve clinical symptoms to a certain extent, but the effect is still not ideal, and the disease recurrence rate is high. As a result, the clinic is paying more and more attention to the role of traditional Chinese medicine in the treatment of pediatric capillary bronchi [5].

The Chinese medicine "Youth Book" records: "Children's lungs are full and full of breath, chest and suffocation, two flaccid movements, trapped into pits, two noses swell, sullen cough... This is the spleen wind," that is, the pediatric capillary bronchi Description of inflammation. Therefore, Chinese medicine believes that children are Shaoyang physique, while those with less yang are vulnerable to injury. If the righteous qi is damaged, the lungs and lungs and spleen bear the brunt. Therefore, the treatment should be based on the principle of nourishing yin and qi, and cultivating soil and producing gold [6]. At present, Chinese medicine treatment of children with bronchiolitis is more common with sputum granules. This product is a proprietary Chinese medicine. The main ingredients are sputum fungus, scorpion and yellow essence, which have the effect of nourishing qi and nourishing yin. And studies have confirmed that Qihuang Granule plays a good role in the treatment of pediatric repeated respiratory infectious diseases, mainly related to the product can enhance the body's immune function and improve the physical condition of children [7]. In addition, based on the pathogenesis of Chinese medicine for bronchiolitis, Shengjin Pingchuan Decoction also has a certain positive effect in its treatment. Shengjin Pingchuan Decoction in Chinese Atractylodes Rhizome, Radix Pseudostellariae is beneficial to lung and spleen, purpura, radish, almond, ephedra, coltsfoot, cough and phlegm, Xuanfei Pingchuan; mustard has warm lungs and phlegm, and clears collaterals and relieves pain The effect, attending chest pain, cold and cough; tangerine peel, Pinellia, phlegm and dampness; phlegm and blood stasis; dry ginger with warming lungs and drinking; platycodon drug loading up the lungs, and Xuanfei sputum function The whole party is well-matched, and plays the role of cultivating earth, gold, phlegm, and asthma. Modern pharmacological studies have confirmed that Shengjin Pingchuan Decoction has the effect of regulating the balance of T lymphocyte subsets and increasing the content of complement $(\mathrm{C} 3, \mathrm{C} 4)$, which is beneficial to improve the immunity of the body. Purpura, coltsfoot flowers, shoots, and platycodon can be used [8].

This study combined with Shengjin Pingchuan Decoction and Qihuang Granule in the treatment of children with bronchiolitis, the results showed that the clinical efficacy, symptoms and signs improved in the observation group after treatment were better than the control group $(\mathrm{P}<0.05)$, see the above combination of drugs can be Effectively improve the therapeutic effect of bronchiolitis, promote the obvious improvement and relief of symptoms and signs, and may be related to the composition of traditional Chinese medicine decoction medicinal materials, which can play a role in multi-target synergistic treatment. The results of this study also showed that the serum TNF- $\alpha$ level in the observation group was lower than that in the control group after treatment $(\mathrm{P}<0.05)$. The objective aspect of the expression of inflammatory factors in the body further confirmed the effective combination of Shengjin Pingchuan Decoction and Qihuang Granule. Bronchiolitis has recurrence characteristics, which can seriously affect the development of children's physical and mental health. Therefore, effectively improving the clinical treatment effect and reducing the disease recurrence rate has always been a key topic for pediatricians. The results of this study showed that after 6 months of follow-up treatment, the recurrence rate of the observation group was $3.77 \%$ lower than that of the control group $(\mathrm{P}<0.05)$, suggesting that the TCM combination regimen is beneficial to reduce the risk of recurrence of the disease, and may regulate the function status of the child with Chinese medicine. It is related to the enhancement of resistance.

In summary, the combination of Shengjin Pingchuan Decoction and Qihuang Granule in the 
treatment of children with bronchiolitis is effective, which can effectively promote the relief of symptoms and signs, which may be related to the down-regulation of serum inflammatory factor expression, and is also conducive to reducing the recurrence rate of the disease.

\section{References}

[1] Sun Haining, He Xiaomin, Wang Guizhi, et al. Effects of Qingjinbi Decoction on lung function and inflammatory factors in children with acute bronchiolitis[J]. Journal of Traditional Chinese Medicine, 2017, 32(2): 190-193.

[2] Qiu Yanzhao, Wu Yonglin. Clinical observation of Qingjin Pingchuan stick in the treatment of acute bronchiolitis in children with phlegm and heat-clearing syndrome [J]. Chinese medicine emergency, 2016, 25(6):1236-1238.

[3] Hu Yamei, Jiang Zaifang. Zhu Fuzhen Practical Pediatrics [M]. 7th ed. Beijing: People's Medical Publishing House, 2003: 1201-1203.

[4] Wang Jingjing, Long Junjie. Diagnostic and therapeutic criteria for clinical symptoms of traditional Chinese medicine [M]. Changsha: Hunan Science and Technology Press, 2006: 261.

[5] Chen Ting, Liu Enmei. Progress in clinical treatment of acute bronchiolitis [J].Journal of Pediatric Pharmacy, 2015, 21(7): 55-58.

[6] Wu Qiuzhen, Feng Bin. Clinical study on the treatment of children with bronchiolitis by integrated traditional Chinese and Western medicine [J]. Journal of Traditional Chinese Medicine, 2017, 32(5): 737-739.

[7] Wang Xia. Effect of Qihuang Granule on the treatment of children with recurrent respiratory tract infection and its effect on cellular immune function[J].Journal of Liaoning University of Traditional Chinese Medicine,2016,18(10):202-204.

[8] Zhang Liyun, Hu Fengqi, Wang Xinying, et al. Clinical control study of Shengjin Pingchuan Decoction in the treatment of children with bronchiolitis[J]. China Medical Guide, 2016, 18(8):805-806. 